\title{
Nuclear Spin Effects in Semiconductor Quantum Dots
}

\section{Citation}

Chekhovich, E. A., M. N. Makhonin, A. I. Tartakovskii, A. Yacoby, H. Bluhm, K. C. Nowack, and L. M. K. Vandersypen. 2013. Nuclear Spin Effects in Semiconductor Quantum Dots. Nature Materials 12, no. 6: 494-504.

\section{Published Version}

doi:10.1038/nmat3652

\section{Permanent link}

http://nrs.harvard.edu/urn-3:HUL.InstRepos:12872193

\section{Terms of Use}

This article was downloaded from Harvard University's DASH repository, and is made available under the terms and conditions applicable to Other Posted Material, as set forth at http:// nrs.harvard.edu/urn-3:HUL.InstRepos:dash.current.terms-of-use\#LAA

\section{Share Your Story}

The Harvard community has made this article openly available.

Please share how this access benefits you. Submit a story.

Accessibility 


\title{
Nuclear spin effects in semiconductor quantum dots
}

\author{
E. A. Chekhovich, M. N. Makhonin, and A. I. Tartakovskii \\ Department of Physics and Astronomy, University of Sheffield, S3 7RH,UK \\ A.Yacoby \\ Department of Physics, Harvard University, Cambridge, MA 02138, USA \\ H. Bluhm \\ 2nd Institute of Physics C, RWTH Aachen University, 52074 Aachen, Germany
}

\author{
K. C. Nowack* and L. M. K. Vandersypen \\ Kavli Institute of Nanoscience, Delft University of Technology, 2600 GA Delft, The Netherlands
}

(Dated: March 28, 2013)

The interaction of an electronic spin with its nuclear environment, an issue known as the Central Spin Problem, has been the subject of considerable attention due to its relevance for spin based quantum computation using semiconductor quantum dots. Independent control of the nuclear spin bath using NMR techniques as well as dynamic nuclear polarization using the central spin itself offer unique possibilities for manipulating the nuclear bath with significant consequences for the coherence and controlled manipulation of the central spin. Here we review some of the recent optical and transport experiments that have explored this central spin problem using semiconductor quantum dots. We focus on the interaction between $10^{4}-10^{6}$ nuclear spins and a spin of a single electron or valence band hole. We also review the experimental techniques as well as the key theoretical ideas and the implications for quantum information science.

Optical and electrical manipulation of single electron and valence band hole spins in semiconductor quantum dots (QDs) has now become possible, owing to the progress in fabrication and new experimental techniques $^{1-8}$, potentially enabling realization of spin qubits for quantum information processing (QIP) ${ }^{9}$. The spin of the confined electron in a QD experiences the hyperfine interaction with $10^{4}-10^{6}$ nuclear spins $^{10-14}$. This interaction is usually quantified using an effective Overhauser magnetic field, $B_{n u c}$, reaching in some cases up to a few Tesla for a highly polarized nuclear spin system ${ }^{12}$, with a statistical fluctuation $\sigma_{B_{n u c}}$ of a few $\mathrm{mT}^{13,14}$. The presence and dynamic properties of the Overhauser field thus have a significant impact on the behavior of the electron spin, and accordingly have received close attention in the quest for realization of a QD spin qubit. Beyond the active research into a quiescent and controllable magnetic environment in solids, nuclear spins themselves have been suggested as a resource with extended coherence (potentially in the ms range) useful for QIP ${ }^{15}$. Very high nuclear polarization degrees now routinely achievable in QDs have also enabled nuclear magnetic resonance (NMR) in single QDs to be realized ${ }^{12,16-18}$, which can be applied for non-invasive probing of chemical composition and strain in the volume occupied by the confined electron ${ }^{19}$.

Here we will review the most pronounced manifestations of nuclear magnetism with a focus on experiments in individual epitaxial and lithographic III-V semiconductor QDs. We will discuss ways to detect and manipulate nuclear spins both in optical and electrical measurements, with one of the 
important goals of reducing the randomness in the nuclear field. We also discuss how the effect of the hyperfine interaction differs for the case of valence band holes compared to electrons. Furthermore, we review NMR experiments in small ensembles of nuclear spins in single QDs. Finally, we will briefly outline imminent future directions in nuclear magnetism research in semiconductor nano-structures.

\section{Hyperfine interaction and detection of nuclear spin polarization}

We start from a brief introduction to electron-nuclear spin interaction, description of typical QD structures, and ways to detect nuclear spin polarization by optical means and electrical probing.

Hyperfine interaction. The dominant contribution to the electron-nuclear hyperfine interaction (HI) originates from the contact Fermi interaction ${ }^{13,14}$ (the hole-nuclear spin interaction is dipole-dipole in nature as described below). The electron-nuclear HI results in a static effect contributing to the energies of the two spin systems, which is usually described in terms of effective magnetic fields: an Overhauser field, $B_{n u c}$, acting on the electron, a result of interaction with a large number of nuclear spins $^{13,14,20}$, and a Knight field experienced by individual nuclear spins as a result of interaction with the spin of the localized electron ${ }^{13,14,16,21}$. Importantly, HI also leads to a dynamical effect responsible for the transfer of spin between the two systems ${ }^{13,14}$.

The nuclear field $B_{n u c}$ fluctuates around its average as a result of the redistribution of nuclear spin polarization due to dipolar coupling or via virtual excitations of the electron spin. In the limit of large $N$, where $N$ is the effective number of nuclei, this can be described by a Gaussian distribution ${ }^{10,11}$ with the standard deviation $\sigma_{B_{n u c}}=B_{n u c}^{\max } / \sqrt{N}$, where $B_{n u c}^{\max }$ is the maximum Overhauser field of the order of a few Tesla ${ }^{22-25}$. For an electron confined in a GaAs quantum dot and interacting with a typical number of $10^{6}$ spin-3/2 nuclei this results in $\sigma_{B_{n u c}} \sim 6 \mathrm{mT}$. $\sigma_{B_{n u c}}$ can exceed $20 \mathrm{mT}$ in small self-assembled $\operatorname{In}(\mathrm{Ga})$ As dots with high concentration of spin-9/2 In. In an experiment with a large number of identical measurements, electron spins initialized in the same state will exhibit different dynamics as they will evolve in a slightly different effective magnetic field. When averaging over many measurements, this will effectively result in spin dephasing on the scale of a typical precession period of the electron in the field of the order of $\sigma_{B_{n u c}}$ : the dephasing time $T_{2}^{*}$ is of the order of $15 \mathrm{~ns}$ for $\sigma_{B_{n u c}} \approx 6 \mathrm{mT}^{1,5,10,11,26,27}$. Much of this dephasing due to the random nuclear field can be unwound using spin-echo techniques, since the nuclear field evolves slowly on the timescale of the electron spin dynamics. The remaining decay of the electron spin coherence, with characteristic timescale $T_{2}$, gives information on the timescale of the nuclear field fluctuations ${ }^{1,5,26,28-30}$.

Detection of nuclear spin polarization in epitaxial quantum dots. We first discuss semiconductor QDs fabricated directly by crystal growth techniques using molecular beam epitaxy (MBE) and metal-organic vapor phase epitaxy (MOVPE) ${ }^{31}$. Structures with typical in-plane dimensions of 20 to 80 $\mathrm{nm}$ and heights of 2 to $10 \mathrm{~nm}$ are formed (Fig.1b), providing strong electron and hole confinement of tens of meV. Of particular interest for spin manipulation in optical experiments are neutral (uncharged) and singly-charged QDs, possible to obtain in charge-tunable devices ${ }^{32}$ or in chemically doped samples ${ }^{33}$.

Detection of nuclear spin polarization is rather straightforward in photoluminescence (PL) of single $\mathrm{QDs}^{20-24,33-37}$. For example, by changing the sign of circular polarization of laser excitation, one can 

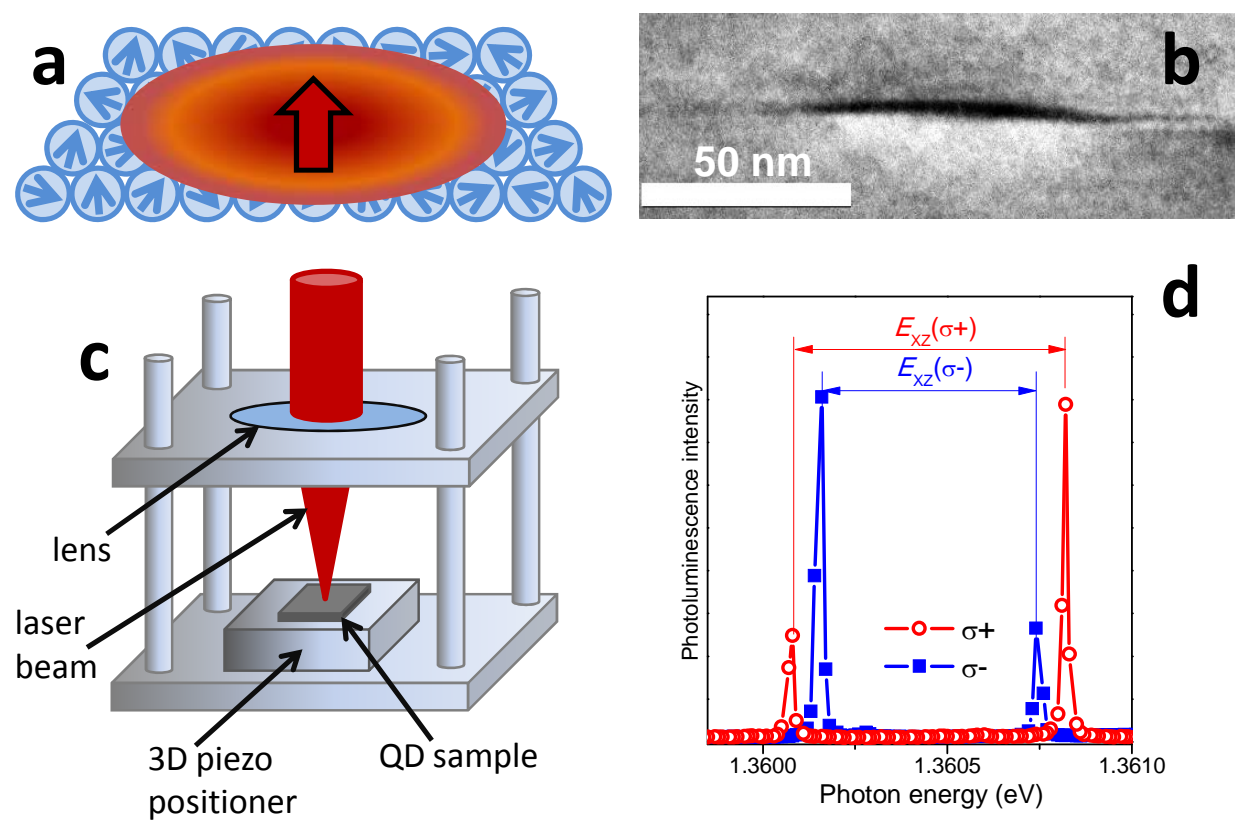

FIG. 1: Optical measurements of nuclear spin effects in quantum dots. (a) A schematic representation of the electron wavefunction in the dot (shown with orange) overlapping with a large number of nuclei (blue circles). Electron spin is shown with a red arrow, and randomly orientated nuclear spins are shown with blue arrows. (b) Transmission electron microscopy image of an InP/GaInP self-assembled quantum dot with darker area corresponding to the In-rich region. (c) A typical micro-photoluminescence ( $\mu$-PL) set-up. A sample is attached to a three-dimensional piezo-positioner enabling its motion with respect to a tight laser spot $(\approx 2 \mu \mathrm{m})$ obtained using a lens with a high numerical aperture. (d) $\mu$-PL spectra measured for single InGaAs/GaAs in external magnetic field $B_{z}=5.3 \mathrm{~T}$ along the QD growth axis, $z$. Circularly polarized non-resonant optical excitation is used. In both cases dynamic nuclear polarization is apparent, as the exciton Zeeman splitting between the peaks in the spectrum measured with $\sigma^{+}$polarized excitation (circles), $E_{X Z}\left(\sigma^{+}\right)$, is larger than that for $\sigma^{-}, E_{X Z}\left(\sigma^{-}\right)$(squares).

easily observe changes in the Zeeman splitting of the QD bright excitons [see Fig.1(d)], reflecting the electron Overhauser shift. In most cases, determination of the absolute degree of nuclear polarization is a difficult task, as it requires accurate knowledge of the QD chemical composition ${ }^{19,22}$. Thus, it is more practical to operate in terms of Overhauser shifts, which can be measured in PL with an accuracy of a few $\mu \mathrm{eV}$, and can be converted to Overhauser fields, $B_{n u c}$, if the electron g-factor $g_{e}$ is known. Similarly to $\mathrm{PL}$, in resonant optical measurements on single dots, such as differential transmission ${ }^{38}$ or resonance fluorescence $^{39}$, the Overhauser shifts can be measured with high accuracy. In measurements on ensembles of QDs, the average degree of nuclear polarization can be extracted either from detailed analysis of PL polarization ${ }^{40}$ or from ultra-fast optical measurements of the Larmor precession of electrons ${ }^{41}$.

Detection of nuclear spin polarization in gate-defined dots. A lithographic QD is formed in a two-dimensional electron gas (2DEG) hosted by a GaAs/AlGaAs heterostructure (Fig.2a) $)^{20,42,43}$. Surface gates on top of the heterostructure are used to locally deplete the 2DEG, which makes it possible to control the electron number in the formed QDs, the tunnel coupling between neighboring QDs, and 
the tunnel coupling between the QDs and reservoirs. Typical dimensions of these dots are $40 \mathrm{~nm}$ in the plane and $10 \mathrm{~nm}$ in the growth direction. Gate-defined dots are probed electrically by either measuring electron transport through the QD (or through several dots in series), or by directly probing the charge state of the QD using a nearby charge detector ${ }^{20,42}$.

Analogously to the case of optical measurements, the nuclear polarization along the external magnetic field $B_{\text {ext }}$ can be probed by measuring the shift induced by $B_{n u c}$ in the total electron Zeeman splitting, $E_{e Z} \cdot E_{e Z}$ can be measured for instance through electron spin resonance $(\mathrm{ESR})^{2,45}$ or electric-dipole spin resonance $(\mathrm{EDSR})^{46,47}$. The width of the resonance peak measured with sufficiently long time averaging gives directly $\sigma_{B_{n u c}}$ in the absence of dynamic nuclear polarization (DNP) and a bound on $\sigma_{B_{n u c}}$ when DNP is present. An alternative to spectroscopic measurements is to directly observe how the time evolution of the electron spin is affected by the nuclear field. For instance, the Larmor precession frequency of a single spin precessing about a static magnetic field is modified by $g_{e} \mu_{B} B_{n u c} / h\left(\mu_{B}\right.$-Bohr magneton, $h$ - Planck constant). If $B_{n u c}$ fluctuates over time, a time-averaged measurement of the electron spin precession will contain a spread of precession rates, leading to decay of the envelope with a time constant $T_{2}^{*}=\hbar \sqrt{2} /\left(g_{e} \mu_{B} \sigma_{B_{n u c}}\right)^{26}$.

$\mathrm{E}(\mathrm{D}) \mathrm{SR}$ or electron spin precession is commonly detected by observing the lifting of the so-called Pauli spin blockade ${ }^{42,48}$ (see Fig.2c). This blockade occurs at the transition between the $(1,1)$ and $(0,2)$ charge regions in a double quantum dot, where tunneling is only allowed for spin-singlet states, but blocked for triplets [(n,m ) refers to $\mathrm{n}$ and $\mathrm{m}$ electrons in adjacent dots]. If $\mathrm{E}(\mathrm{D}) \mathrm{SR}$ flips the spin of one of the electrons of a triplet, the blockade is lifted. Pauli blockade is also directly used to access the average $B_{n u c}$, the difference of the nuclear field in the two dots, $\Delta B_{n u c}$, and the uncertainties in these two quantities. For instance, the blockade is lifted at a crossing of two states with different spin, where electron-nuclear flip-flops mix the spin states without energy cost. Hence, the detuning $\epsilon$ at which the $\mathrm{m}=1$ triplet $T_{+}$crosses the singlet branch $\mathrm{S}$ (see Fig. $2 \mathrm{~d}$ ) ${ }^{49}$ can be detected, which depends on $E_{e Z}=g_{e} \mu_{B}\left(B_{e x t}+B_{n u c}\right)$. Hence $B_{n u c}$ can be extracted at a given $B_{\text {ext }}$. Another example is to start from the singlet ground state at large positive detuning and initiate oscillations between $S$ and $T_{0}$ (the $\mathrm{m}=0$ triplet) with frequency $f=\left|g_{e} \mu_{B} \Delta B_{n u c}\right| / h$ by a fast gate voltage pulse to large negative detuning. The decay time of the oscillation gives a measure of $\sigma_{\Delta B_{n u c}}$. Using single shot readout, such a measurement of $\Delta B_{n u c}$ involving thousands of samples takes less than $10 \mathrm{~ms}^{50,51}$ allowing real time tracking of $\Delta B_{n u c}$ (Fig.2e). The time resolution is sufficient to resolve the Overhauser field fluctuations resulting in a detailed picture of the spin dynamics.

\section{Dynamic nuclear polarization}

The HI enables not only sensing of the nuclear magnetic field through measurement of the electron spins, but also manipulation of the nuclear spins via the electron spins: The transverse terms of the HI mediate electron-nuclear flip-flops ${ }^{13,14}$ in which the electron changes its spin by \pm 1 with a simultaneous change of the spin of one of the nuclei by $\mp 1$. These flip-flop terms generate dynamic nuclear polarization (DNP) when the spin pumping rates up versus down are asymmetric. A steady-state nuclear spin polarization is reached when nuclear spin pumping rates are balanced by nuclear spin relaxation rates. Under most conditions, DNP is weak since nuclear spin pump rates are suppressed due to the mismatch between the nuclear and electron Zeeman splitting. However, there are a number 
a

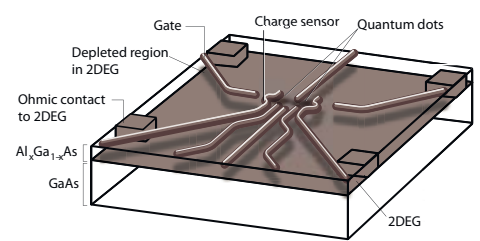

b

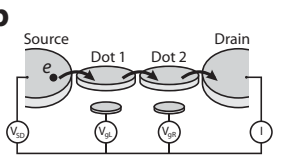

C
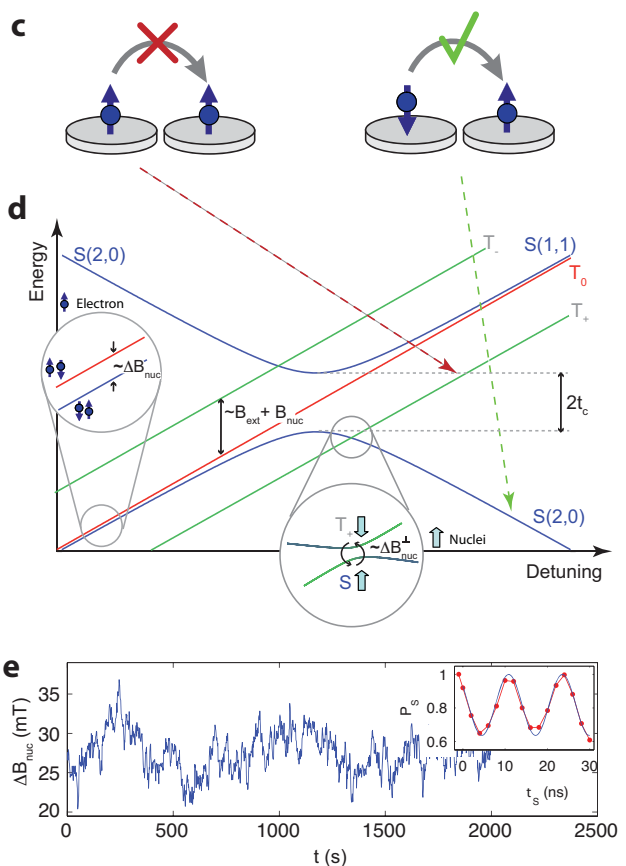

FIG. 2: Electrical probing of nuclear spin effects in gate-defined quantum dots. (a) Schematic of a gate-defined double QD (adapted from Ref. ${ }^{43}$ ). (b) Measurement of the current through a double QD (left) and sensing of the occupation of each dot using a nearby charge detector (right). (c) Principle of Pauli spin blockade used to convert spin to charge information in a double QD. The Pauli exclusion principle forbids electrons with parallel spins (spin triplet) to occupy the same dot (left), whereas double occupancy is allowed for electrons with anti-parallel spins (spin singlet, right). (d) Energy levels of a double QD as a function of the relative energy detuning, $\epsilon$, between the $(1,1)$ and $(0,2)$ charge configurations. $\epsilon$ can be controled through the voltages applied to the gates (see $V_{g L}$ and $V_{g R}$ in (b)). $S(1,1)$ and $S(2,0)$ denote the spin singlets in $(1,1)$ and $(2,0)$. Due to the Pauli exclusion principle, the only relevant state in the $(0,2)$ region is $S(0,2)$. Near the transition, the $S(1,1)$ and $S(2,0)$ singlet states hybridize due to the inter-dot tunnel coupling $t_{c} . T_{+}=|\uparrow \uparrow\rangle, T_{0}=\frac{1}{\sqrt{2}}(|\uparrow \downarrow\rangle+|\downarrow \uparrow\rangle)$, and $T_{-}=|\downarrow \downarrow\rangle$ are the three $(1,1)$ triplets with magnetic quantum number $\mathrm{m}=+1,0$, and -1 . $T_{+}$and $T_{-}$split off due to $B_{\text {ext }}$. Far left in the $(1,1)$ region, the eigenstates turn into $|\uparrow \downarrow\rangle$ and $|\downarrow \uparrow\rangle$ because of the difference of $B_{n u c}$ in the two dots, $\Delta B_{n u c}$ (left inset). The degeneracy point of $S=\frac{1}{\sqrt{2}}(|\uparrow \downarrow\rangle-|\downarrow \uparrow\rangle)$ and $T_{+}$(middle inset) can be used for polarizing nuclear spins. (e) Time trace of $\Delta B_{n u c}$. Each data point reflects the frequency $g \mu_{B} \Delta B_{n u c} / h$ of an oscillation between $\mathrm{S}$ and $T_{0}$ (inset) . Data from Ref. ${ }^{44}$. 
of ways to overcome the energy mismatch and to induce a preferential pumping direction, both by optical and electrical means. There have been many attempts to find an efficient way of achieving DNP $^{21-25,33,34,49,52,53}$, with the main motivation to achieve a $100 \%$ polarization of nuclear spins, which would prevent nuclear-nuclear flip-flops, strongly suppressing the randomness in the nuclear field and concomitant electron spin decoherence ${ }^{10}$.

Dynamic nuclear polarization in optically pumped quantum dots. Most experiments on optical pumping of nuclear spins in QDs are performed at temperatures below $50 \mathrm{~K}$ (normally below 10 $\mathrm{K})$. In most cases DNP occurs following the spin transfer from optically pumped or resident electrons. Electron Overhauser shifts in excess of $100 \mu \mathrm{eV}$ can be obtained using optical pumping ${ }^{22-25,33,34}$, and $B_{\text {nuc }}$ up to $3 \mathrm{~T}$ have been reported ${ }^{23-25}$. Using rough estimates of the dot composition, degrees of nuclear polarization up to $60 \%$ are now routinely obtained.

DNP is readily observed under excitation of QDs with circularly polarized light ${ }^{21-25,33-38,54,55}$ : when $\sigma^{+}$or $\sigma^{-}$polarized photons are absorbed by the sample, electrons with well-defined spin orientation may be created ${ }^{14,56}$. This still holds for so-called 'non-resonant' excitation when the laser is tuned up to $100-200 \mathrm{meV}$ above the QD lowest energy levels ${ }^{21-25,34,54}$. Very efficient nuclear spin pumping also occurs under resonant excitation into the lowest energy states of the $\operatorname{dot}^{33,38,39,57,58}$. This leads to various "line-dragging" effects as the excitation laser is tuned seemingly out of resonance but build up of the Overhauser field maintains the on-resonance condition ${ }^{33,38,57-59}$.

The efficiency of electron-nuclear spin flip-flops leading to DNP depends on the energy splitting between the initial and final electron (exciton) states involved in the electron spin-flip, $\Delta E_{\uparrow \downarrow}$, and scales roughly as $1 / \Delta E_{\uparrow \downarrow}^{2} . \Delta E_{\uparrow \downarrow}$ may be as large as $0.1-0.5 \mathrm{meV}$, and is a major energy cost of the flip-flop process. Owing to the requirement of the energy conservation, in most cases the spin flip-flop occurs as a second order process: the electron is virtually transferred to the state with the opposite spin, while a single nuclear spin is flopped; the electron then escapes from the dot (or a trion is formed in a charged dot), the process usually accomplished by emission (absorption) of a photon ${ }^{21-25,33-38,54,55}$ or electron tunneling from the $\operatorname{dot}^{55,57,58}$. Note that $\Delta E_{\uparrow \downarrow}$ is dependent on both $B_{e x t}$ and $B_{n u c}$. This gives rise to an intrinsic feedback mechanism in the DNP process, which is a source of pronounced bi-stabilities and switching of nuclear polarization in a QD under optical pumping ${ }^{23,24,35}$ (Fig.3a).

Dynamics of the optically induced spin pumping have been studied in pump-probe experiments ${ }^{36,38,61-63}$. For small $B_{\text {ext }}$ (i.e. small $\Delta E_{\uparrow \downarrow}^{2}$ ), nuclear polarization build-up time under optical pumping is in the millisecond range ${ }^{36,61}$, whereas in higher fields of a few $\mathrm{T}$, the typical build-up time is of the order of 1-10 $\mathrm{s}^{38,61,63}$. Nuclear spin depolarization can occur via flip-flops between interacting (neighboring) nuclei, giving rise to nuclear spin diffusion. However, in most QDs the nuclear Zeeman splitting matching required for such nuclear-nuclear flip-flops is not fulfilled owing to quadrupole interactions occurring mainly as a result of strain ${ }^{13,19,60,64-66}$. Thus nuclear spin diffusion is typically suppressed in $\mathrm{QDs}^{36,60,61,63}$. The presence of strain and hence strong quadrupole effects is one of the main reasons that DNP is possible in zero external magnetic field ${ }^{21,33,36,61,67}$, where nuclear polarization life-times in the dark of up to tens of seconds have been observed ${ }^{36,61}$. Remarkably, in magnetic fields of a few $\mathrm{T}$, nuclear polarization in strained self-assembled dots survives as long as 30 hours ${ }^{36,60,61}$. On the contrary, if a $\mathrm{QD}$ is brought in contact with an unpolarized electron reservoir, as is often the case in charge-tunable devices, nuclear spins are depolarized in a few milliseconds ${ }^{36}$. 

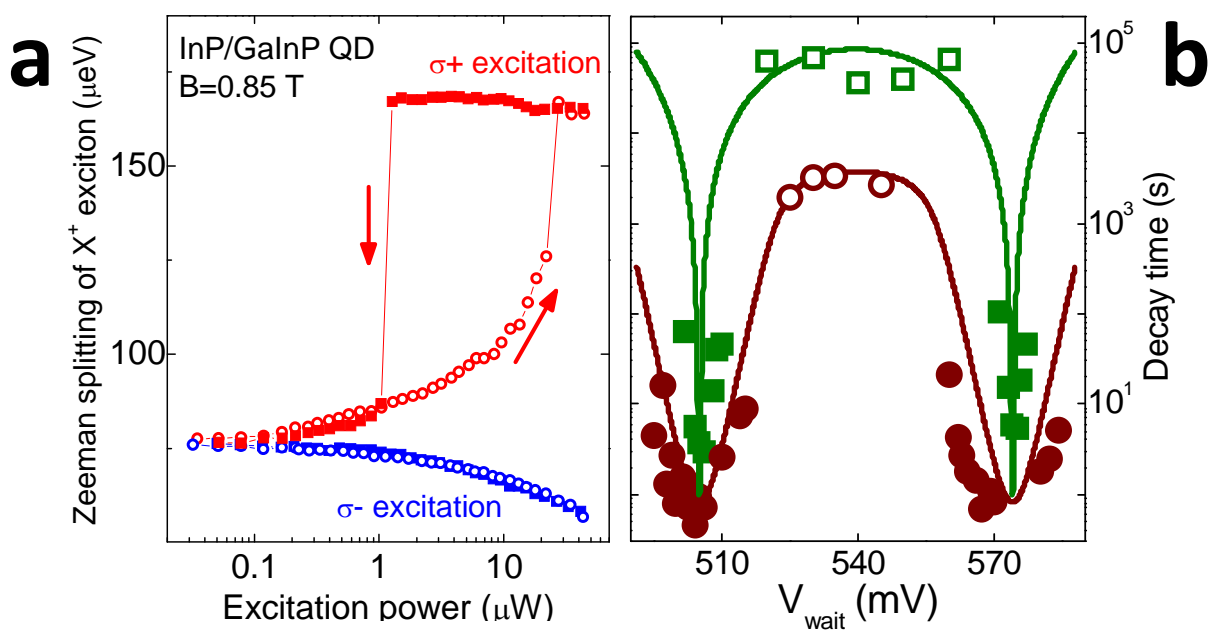

FIG. 3: Dynamic nuclear polarization (DNP) in optically pumped quantum dots. (a) Bistable behavior of nuclear polarization in a positively charged InP/GaInP quantum dot pumped non-resonantly with circularly polarized light in external magnetic field along the growth axis $B_{z}=0.85 \mathrm{~T}$. The plot shows the Zeeman splitting of the positively charged exciton, $X^{+}$, measured in photoluminescence as the laser excitation power is scanned from high to low and back. Directions of the scans are shown with arrows for $\sigma^{+}$pumping, inducing Overhauser field $B_{n u c}$ (felt by optically excited electrons) anti-parallel to $B_{z}$ and leading to the reduction of the electron Zeeman splitting. This causes positive feedback for DNP and results in switching and bistability of $B_{n u c}{ }^{23,24,35}$. In contrast, under $\sigma^{-}$pumping, $B_{n u c}$ is parallel to $B_{z}$, causing the slow-down of DNP as $B_{n u c}$ increases. (c) Nuclear spin polarization decay times in an InGaAs QD in a Schottky diode as a function of bias measured at temperatures of $4 \mathrm{~K}$ (dark red) and $0.2 \mathrm{~K}$ (green) and $B_{\text {ext }}=5 \mathrm{~T}$. The decay of the Overhauser field is mediated by the electron cotunneling between the dot and the electron reservoir in the contact, particularly pronounced at the edges of the charging plato around 505 and $575 \mathrm{mV}$ in this graph. The decay time increases up to $10^{5} \mathrm{~s}$ for $T=0.2 \mathrm{~K}$ at biases away from the cotunneling regime. Data in (b) from Ref. ${ }^{60}$.

Dynamic nuclear polarization in gate-defined quantum dots. The first observation of DNP in gated quantum dots was reported in 2004 by Ono et al. ${ }^{68}$ manifesting itself in hysteretic magnetic field sweeps (Fig. 4a) and slow oscillations of the leakage current through a double quantum dot in the spin blockade regime. In the same regime, several experiments report hysteresis ${ }^{52,69}$ and bistable current behavior $^{69}$. Polarization up to $\approx 40 \%$ has been claimed $^{52}$. The complexity of the interplay between nuclear spin polarization and transport in the spin blockade regime has triggered a large amount of theoretical work ${ }^{70-74}$. Although in the experiments many details of the observed behavior are not understood, all have in common that a degeneracy of two-spin states with different spin, such as $S$ and $T_{+}$(the $m=1$ triplet) and $S$ and $T_{-}$(the $m=-1$ triplet) appears to be the main origin of electronnuclear flip-flops (see Fig. 4d). At these crossings electron-nuclear flip-flops are possible at no energy cost.

Compared to these transport measurements, experiments with specially designed gate voltage pulse cycles offer a more controlled way to realize a DNP pump scheme. In Ref. ${ }^{49}$, the system is first initialized in the $S(0,2)$ state and subsequently, an adiabatic sweep across the $S-T_{+}$degeneracy point (see Fig. $2 \mathrm{~d}$ 

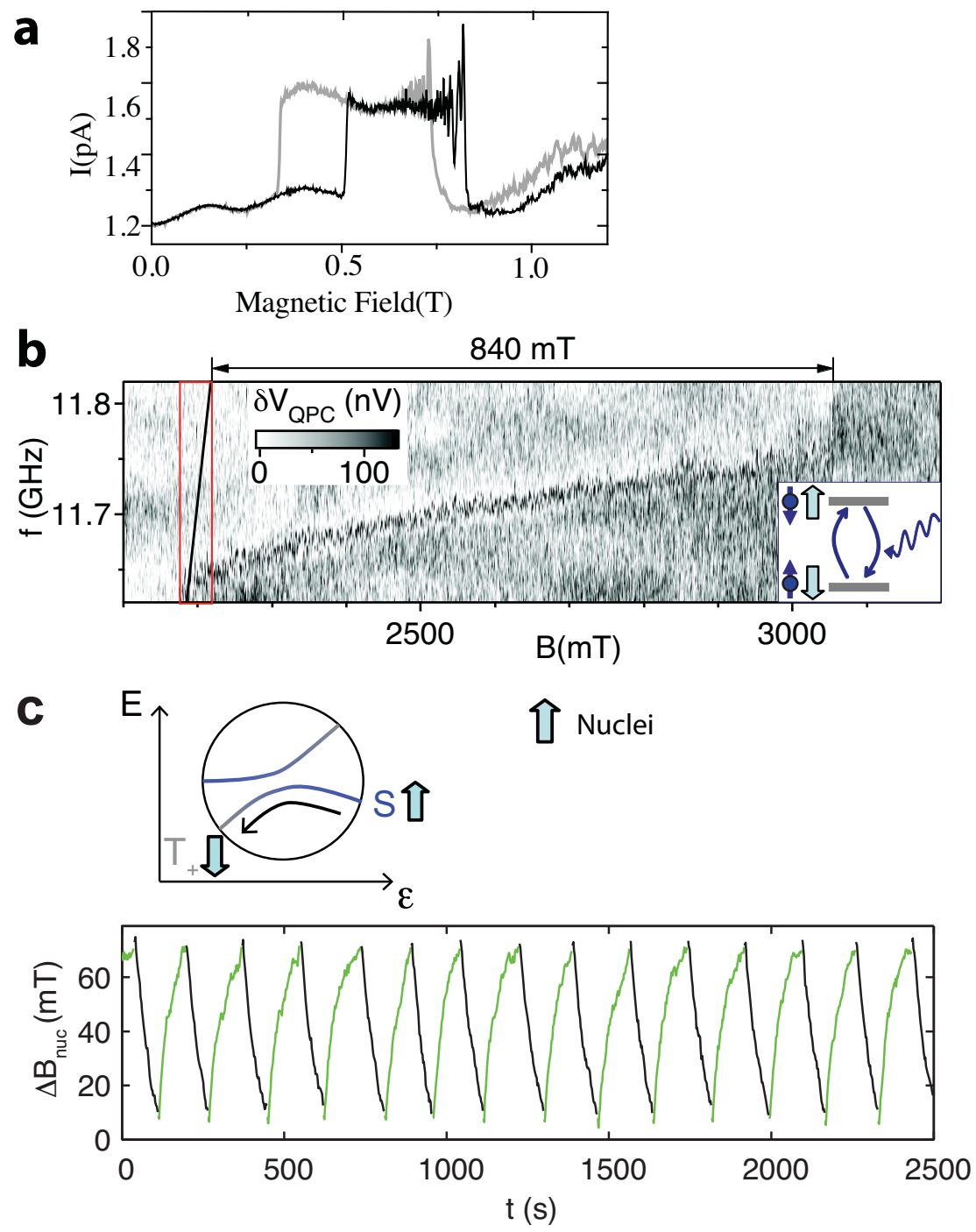

FIG. 4: Dynamic nuclear polarization in gate-defined quantum dots. (a) DNP leads to a hysteretic leakage current in the spin blockade regime as a function of increasing and decreasing magnetic field. Data from $^{68}$. (b) Average charge occupation of a double quantum dot in the spin blockade regime under the influence of an ac electric field. When the excitation frequency $f$ is resonant with the electron Zeeman splitting, it drives electron-nuclear flip flops (inset), thus lifting the spin blockade and changing the average occupation (darker regions). As the field $B_{\text {ext }}$ (directed along $z$ ) is swept upwards, a nuclear polarization partly counteracts the change of $B_{\text {ext }}$, thus moving the resonance away from its equilibrium position (black diagonal line) by up to 840 mT. Data from ${ }^{47}$. (c) Control of the hyperfine field gradient in a double quantum dot operated as an $S-T_{0}$ qubit. DNP is obtained by sweeping the detuning through the $S-T_{+}$transition (top), causing spin transfer between electrons and nuclei. Each data point on the lower panel reflects a measurement of $\Delta B_{n u c}$ as shown in the inset to Fig. 2e. DNP pulses were applied between successive measurements. They increase or decrease $\Delta B_{n u c}$ depending on whether the DNP cycle starts from an $S$ (green) or $T_{+}$state (black). Data from Ref. ${ }^{44}$ 
and 4c) transfers up to one unit of angular momentum into the nuclear spin bath. Finally, one electron is pushed out of the double dot and the next cycle begins. Spin transfer in the opposite direction and thus full bidirectional control (Fig. 4c) was demonstrated ${ }^{53}$ by initialization of a $T_{+}(1,1)$ state followed by a similar slow passage through the $S-T_{+}$degeneracy point. These pump cycles can be extended to reduce fluctuations of the hyperfine field ${ }^{44,45}$.

Rather than exploiting level degeneracies, the energy for electron-nuclear flip-flops can be provided by a resonant ac electric field (inset to Fig. 4b). The electric field modulates the hyperfine coupling constant of each nucleus to the electron and therefore the transverse term of the hyperfine coupling. This directly drives electron-nuclear flip-flops ${ }^{45,47}$ (see Fig.4b).

We will now discuss nuclear spin dynamics in gate-defined QDs, which can be measured accurately using fast manipulation of the electron spin by gate voltages. Here, two different pictures of the nuclear spin dynamics are useful. At short times, the fluctuations of the Overhauser field are determined by the $\mathrm{HI}$ and the nuclear Larmor precession, whereas at longer times $\left(>10^{-4} \mathrm{~s}\right)$, nuclear flip-flops due to the dipolar coupling lead to a diffusion-like redistribution of the local nuclear polarization inside and outside the quantum $\operatorname{dot}^{11}$.

The diffusive long-time behavior has been probed by directly measuring the fluctuations of the Overhauser field ${ }^{75,76}$ using methods discussed above (see Fig.5a). At low magnetic fields $(\lesssim 20 \mathrm{mT}$ ), one finds an about tenfold speedup of spin diffusion ${ }^{75}$ that likely reflects the activation of additional diffusion channels by the reduced Zeeman energy mismatch, such as electron mediated spin transfer between nuclei. The electron mediated diffusion also leads to a dependence of the decay rate of an induced polarization on the occupancy of the $\operatorname{dot}^{76}$. Note, that gate-defined QDs are usually made of unstrained GaAs, therefore quadrupole effects are weak and were neglected in the discussion above.

The short-time nuclear spin dynamics have been probed via electron spin dephasing under inversion of the electronic state halfway though an interval of free evolution ${ }^{77}$, a procedure known as Hahnecho. This technique is only sensitive to the relatively fast changes of the Overhauser field during the evolution. Measuring the final electronic state gives detailed insight into the nuclear spin dynamics on the microsecond time scale ${ }^{28}$ (see Fig. 5b,c). The monotonic decay of the Hahn-echo signal with characteristic evolution time, $\tau$, at high fields is a result of the diffusive dynamics of $B_{n u c}^{z}$ due to dipolar coupling. The resulting spectral diffusion is predicted to cause a $\exp \left(-\left(\tau / T_{S D}\right)^{4}\right)$ decay of the echo ${ }^{78,79}$ with a characteristic time constant $T_{S D}$ of a few tens of $\mu \mathrm{s}$.

The oscillations found at lower fields, which eventually turn into full collapses and revivals, were first predicted based on a fully quantum mechanical treatment ${ }^{73}$, but can also be understood with a semiclassical model. It is based on the electronic Zeeman energy splitting being proportional to the total magnetic field $B_{t o t}=\sqrt{\left(B_{\text {ext }}+B_{\text {nuc }}^{z}\right)^{2}+B_{\text {nuc }}^{\perp}} \approx B_{\text {ext }}+B_{\text {nuc }}^{z}+B_{\text {nuc }}^{\perp} / 2 B_{\text {ext }}$ (Fig. 5c top left). Dephasing is caused by fluctuations of this level splitting and thus related to the time-dependence of both the parallel and transverse nuclear components, $B_{\text {nuc }}^{z}$ and $B_{\text {nuc }}^{\perp}{ }^{2}$. The collapses and revivals observed in the electron spin echo signal arise from the phase associated with $B_{\text {nuc }}^{\perp}{ }^{2}$. The transverse nuclear field, $\vec{B}_{\text {nuc }}^{\perp}$, is a vector sum of contributions from the three nuclear species ${ }^{69} \mathrm{Ga},{ }^{71} \mathrm{Ga}$ and ${ }^{75} \mathrm{As}$ (Fig. 5c, top right). Due to the different precession rates of these species, $B_{\text {nuc }}^{\perp}{ }^{2}$ thus oscillates at the three relative Larmor frequencies (Fig. 5c, bottom). The amplitude and phase of the oscillating nuclear fields fluctuate over the course of many repetitions, thus leading to randomization of the resulting phase and suppression of the echo signal. However, if the precession interval is approximately a multiple of all 

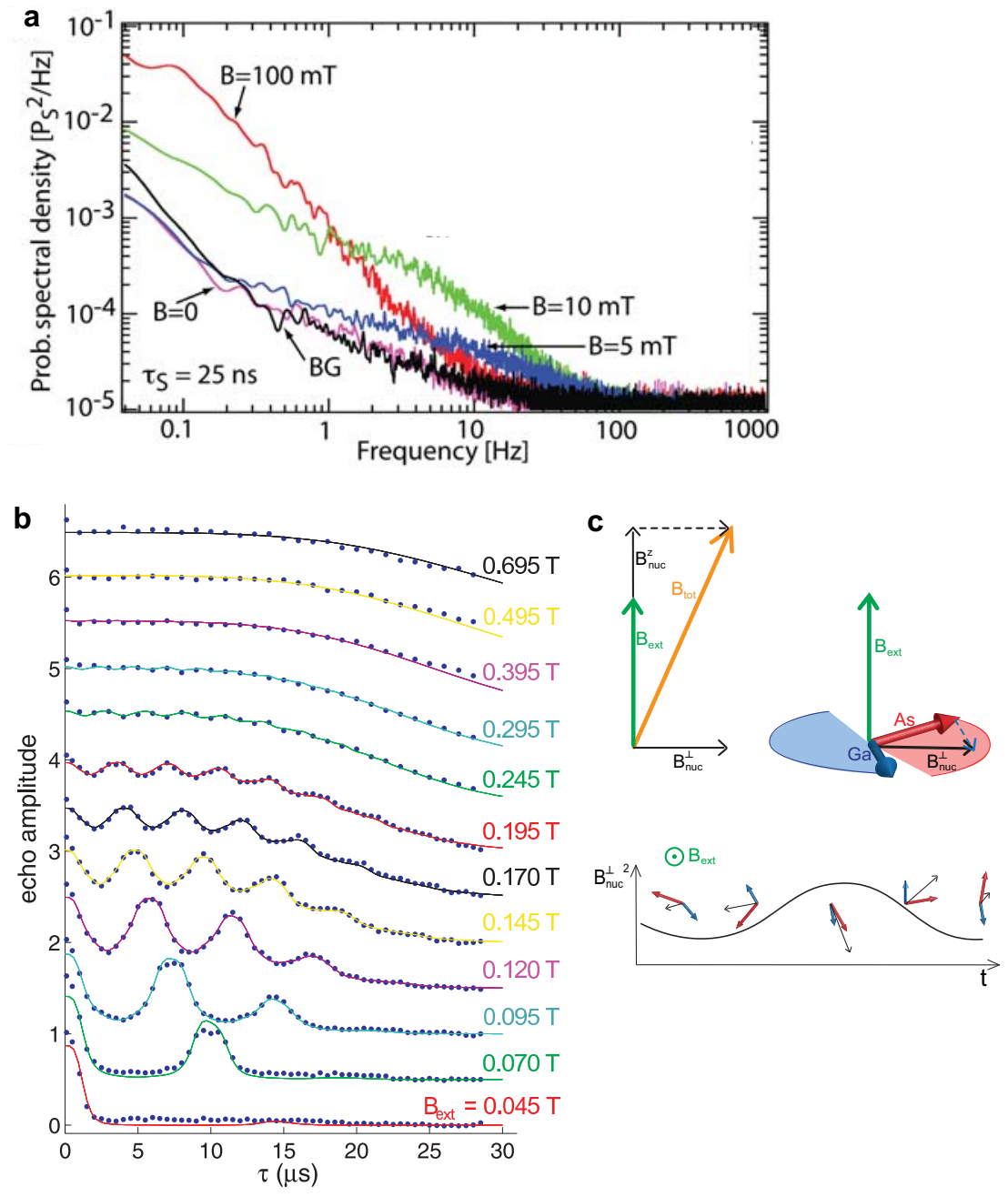

FIG. 5: Dynamics of nuclear spins in a gated double-dot structure. (a) Spectra of the fluctuation of the nuclear hyperfine field at relatively low frequency, obtained from time traces of the singlet probability of the qubit after precession in the Overhauser field over a fixed evolution time $\tau_{S}$. A significant speedup of the dynamics is observed at low magnetic fields. The shape of the spectrum can be explained in terms of nuclear spin diffusion. (b) Hahn echo signal in a $S-T_{0}$ qubit as a function of the total evolution time, $\tau$, for different values of magnetic field. Exchanging the two electrons at time $\tau / 2$ via a gate voltage pulse causes them to see the same static hyperfine field, so that only fluctuations during $\tau$ reduce the probability of the electrons to return to their initial state, which is reflected in the echo amplitude. Curves are offset for clarity and normalized. Data are shown as dots, fits as solid lines. (c) Illustration of the the semiclassical model used for the fits (see main text). Adapted from Refs. ${ }^{75}$ (a) and $^{28}$ (b, c)

three Larmor periods, the oscillations imprint no net phase on the electron spin and the echo amplitude revives. A quantitative model treating the components of $\vec{B}_{\text {nuc }}^{\perp}$ as classical variables also explains the faster decay of the echo envelope at low fields (Fig. 5b) in terms of dephasing of the nuclear spins themselves.

\section{Interaction of valence band holes with nuclear spins}




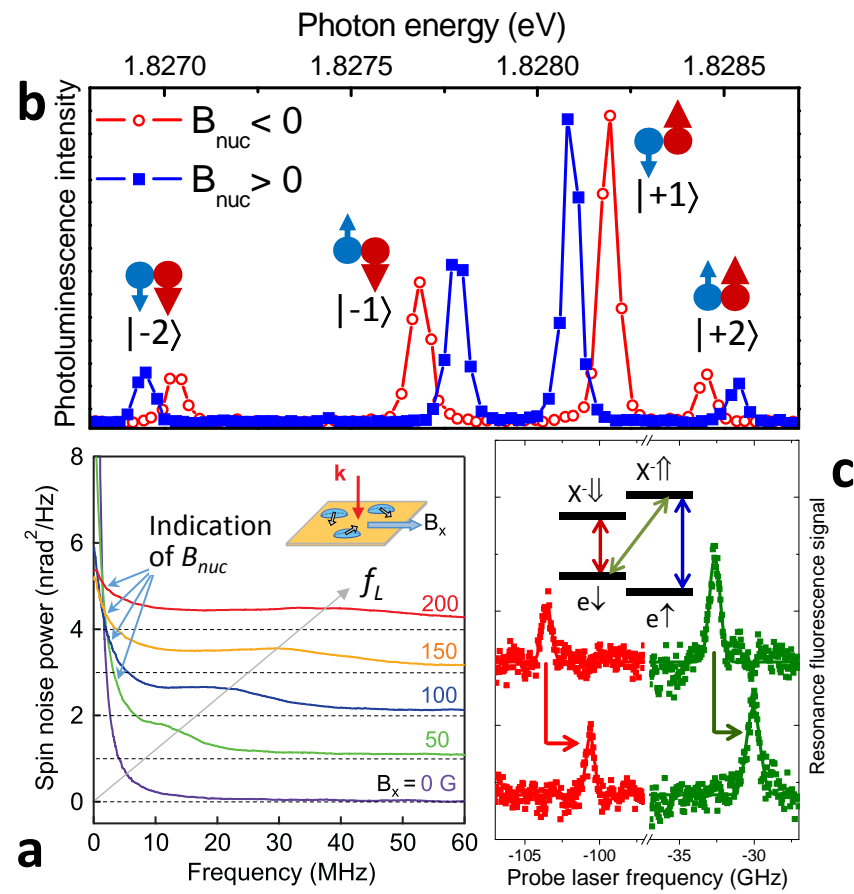

FIG. 6: Hole-nuclear spin interaction in optically pumped quantum dots. (a) Hole spin noise in transverse applied magnetic fields $\left(B_{x}\right)$. A finite noise component remains at zero frequency. This reveals the presence of the longitudinal (along $z$ ) components of the nuclear Overhauser magnetic field acting on holes. (b) Photoluminescence spectra of a single neutral InP/GaInP QD at $B_{z}=6 \mathrm{~T}$ for negative (blue) and positive (red) nuclear spin polarization induced optically on the dot. Bright $(| \pm 1\rangle)$ and dark $(| \pm 2\rangle)$ excitons are observed and the corresponding spin orientations of the electron (blue) and hole (red) are shown schematically. The change in the splitting between the bright $|+1\rangle$ and dark $|+2\rangle(|-2\rangle)$ states corresponds to the hole (electron) Overhauser shift. By comparing these shifts, the ratio between the electron and hole hyperfine constants can be extracted. (c) Inset: laser excitation scheme for measurements of the hole hyperfine constant in a negatively charged dot in magnetic field. Three lasers are shown: (i) polarizing nuclei via an allowed transition and driving the resonance fluorescence (blue); (ii) probing the other allowed transition (red); (iii) probing weakly allowed transition (green). When "red" or "green" lasers are on resonance with the e- $X^{-}$transitions, resonant enhancement of fluorescence is detected shown with red and green symbols, respectively. The hole (electron) hyperfine shift can be extracted from the difference between (sum of) the shifts of the "red" and "green" peaks as the nuclear polarization on the dot is varied by adjusting the frequency of the blue laser. The two sets of data at the top and bottom of the plot are measured for two different degrees of nuclear polarization on the dot. Figures are adapted from Ref. ${ }^{80}$ in (a), Ref. ${ }^{37}$ in (b), and Ref. ${ }^{39}$ in (c). 
Unlike electrons having $s$-type atomic wavefunctions, the hole has a wavefunction constructed predominantly from $p$-orbitals with zero density at the nuclear site. This leads to a vanishing contact part of the $\mathrm{HI}$, which combined with extended hole spin life-times in $\mathrm{QDs}^{81}$ presents holes as a potentially viable alternative to electrons for implementation of spin qubits ${ }^{81,82}$. Recent theory predicts that the hole HI, dipole-dipole in nature, can be as large as $10 \%$ of that of the electron, and is strongly anisotropic ${ }^{83-86}$. Furthermore, heavy-hole (hh) states with pure $p$-symmetry couple only to the nuclear field along $z$, i.e. exhibit an Ising-type interaction with nuclear spins and slow decoherence ${ }^{84,85}$. On the other hand, it has been shown theoretically that the HI leads to efficient decoherence of the pure hh states having an admixture of $d$-orbitals in the wave-function, estimated to be considerable (e. g. $\sim 20 \%$ for Ga) from recent experiments ${ }^{87}$. Another decoherence mechanism arises from heavy-light hole mixing, as light-hole (lh) states couple to all nuclear spin components ${ }^{84,86}$. However, in the majority of studied QDs hh-lh mixing is very small ${ }^{81,82}$, so this decoherence mechanism should in principle manifest itself in rare cases ${ }^{84,86,88}$, whereas the contribution of $d$ orbitals is common for III-V semiconductors and will play an important role in a broad class of III-V nano-structures ${ }^{87}$.

Experimental evidence for the hole HI can be obtained from the measurements of the hole spin dynamics. Ensembles of p-doped self-assembled InGaAs/GaAs QDs were studied in low longitudinal magnetic fields $B_{z}$ using ultra-fast optical pump-probe measurements ${ }^{86,88}$ and spin noise spectroscopy ${ }^{80}$. Suppression of the hole spin dephasing in Ref. ${ }^{86,88}$ and sharp increase of the hole spin correlation times in Ref. ${ }^{80}$ was found above a characteristic $B_{z}=2.5-3 \mathrm{mT}$. This gives an estimate of the magnitude of the fluctuating transverse nuclear field acting on the hole spin, about an order of magnitude lower than that for electrons in similar QD samples ${ }^{88}$. The presence of the longitudinal nuclear field can also be evidenced using spin noise detection ${ }^{80}$ [Fig.6(a)].

Measurements on individual QDs using optical detection with high spectral resolution enabled the hole Overhauser shift to be measured directly and simultaneously with that for the electron ${ }^{33,39}$. The ratio between the all-element-averaged hole $(C)$ and electron $(A)$ hyperfine constants was found to be $C / A \approx-0.1 \mathrm{in} \mathrm{InP}$ and InGaAs QDs ${ }^{33,39,87}$. Further studies combining optical and radio-frequency (rf) techniques revealed that the $|C / A|$ ratio can reach as high as $0.15-0.2$, and that $C>0$ for anions $(A s$, $P$ ) and $C<0$ for cations $(I n, G a)^{87}$. The sign difference was explained by the contribution of atomic $d$ orbitals to the cationic hole Bloch wavefunction, whereas for anions the wavefunctions is purely $p$-type.

It must be noted, that understanding of the hole spin decoherence and the role of the hole hyperfine interaction may still be incomplete. There is a rather large spread of measured $T_{2}^{*}$ for the hole spin: $>100 \mathrm{~ns}$ using coherent population trapping ${ }^{3}$ and 2 to $20 \mathrm{~ns}$ in ultra-fast optical measurements of the hole spin Ramsey fringes ${ }^{6-8}$. It is also an emerging paradigm that electrical noise in the diodes comprising hole-charged QDs may be a factor strongly limiting the $T_{2}^{*}$ values ${ }^{6,7}$. On the other hand, the more fundamental property, such as the admixture of $d$-orbitals in the hole Bloch function ${ }^{87}$ important for hole spin decoherence via the HI, may also vary from dot-to-dot leading to variation in $T_{2}^{*}$ values.

\section{Narrowing of nuclear field distribution using 'closed-loop' spin pumping}

The preceding sections discussed experiments where angular momentum is transferred from the local electron spin to the nuclear spin bath in an open-loop mode. As a result, the nuclear field was always 
subject to statistical fluctuations. Here we present a development of the last few years, realized by both optical and electrical means, which is closed-loop control of the nuclear field, suppressing its randomness to well below the statistical fluctuations. The suppression of the bath fluctuations immediately leads to extended dephasing times and better control of the time evolution of the central spin.

Suppression of nuclear spin fluctuations using optical pumping with feedback. In optically pumped self-assembled dots, such suppression of nuclear spin fluctuations using "active" stabilization has been achieved by resonant $\mathrm{cw}^{27,38}$ and pulsed ${ }^{41}$ laser excitation. The stabilization was achieved via feedback reversing any changes in the nuclear polarization so that the QD optical transition remained in resonance with the $\mathrm{cw}$ laser ${ }^{27,38}$, or spin precession of the QD electron remained synchronized with the pulse repetition rate of the ultra-fast laser ${ }^{41}$. In all of these experiments, low or moderate degree of nuclear spin polarization was achieved, in contrast to the previously considered requirement of nearly $100 \%$ nuclear polarization degree in order to suppress nuclear spin fluctuations ${ }^{10}$.

Greilich et al. ${ }^{41}$ used Faraday rotation (FR) in ensembles of electron-charged self-assembled InGaAs dots. Electron spin precession in a large number of dots becomes synchronized with the repetition rate of the laser by means of DNP and despite the initial ensemble spread of the electron g-factors. Deviation from the synchronization condition caused, for example, by nuclear spin fluctuations, would lead to increased light absorption in the dot, which in turn lead to nuclear spin pumping until the nuclear spin projection along the in-plane external field was restored to fulfill the synchronization $\operatorname{condition}^{89}$.

Latta et al. ${ }^{38}$ presented measurements showing the damping of fluctuations in the optical transition energy in a single QD by locking the quantum-dot resonance to the incident laser. The locking was achieved by dynamic nuclear polarization, which also resulted in the "line-dragging" and a marked distortion of the QD line-shape in differential transmission ${ }^{38}$. As the model simulations show, the optical transition stabilization occurring as a result of DNP is accompanied with a significant narrowing of the Overhauser field variance.

$\mathrm{Xu}$ et al. ${ }^{27}$ observed enhancement of the electron $T_{2}^{*}$ using coherent dark-state spectroscopy carried out on a single electron-charged dot. This effect was explained by suppression of nuclear spin fluctuations under the Overhauser field locking similar to the line-dragging in Ref. ${ }^{38}$. A marked enhancement of the electron $T_{2}^{*}$ by a factor of several hundred, arising as a result of suppressed nuclear spin fluctuations, was observed.

Narrowing in gate defined dots. In gate-defined dots, several quite distinct approaches to suppress nuclear field fluctuations have been successfully used. The conceptually simplest possibility for suppressing the randomness of the Overhauser field (or its gradient) is to rapidly measure it and to use DNP to restore its desired value. For $S-T_{0}$ qubits, this approach ${ }^{4}$ permitted a reduction of the rmsfluctuations of the hyperfine field gradient, $\sigma_{\Delta B_{n u c}}$, by about a factor of 2 . A more powerful approach relies on directly conditioning the spin transfer from the electrons to the nuclei on the current value of the hyperfine field, thus letting the electron spin itself act as a complete feedback loop not requiring external intervention. This approach was used to control both the hyperfine field in the individual halves of a double quantum dot using an ESR-based spin transfer technique ${ }^{45}$, and the field gradient $\Delta B_{n u c}$ between the two dots of an $S-T_{0}$ qubit via exchange mediated spin transfer ${ }^{44}$.

Such feedback schemes can be understood based on the so called pumping curve, which provides the 
a

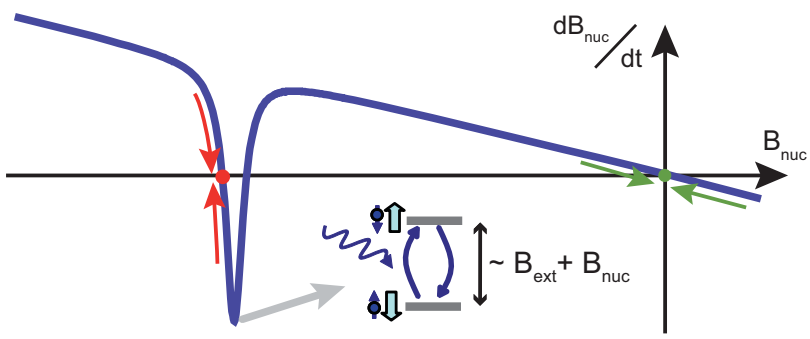

b

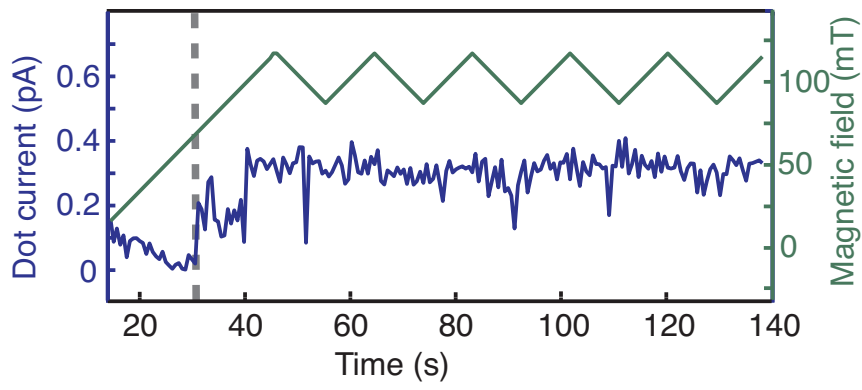

C
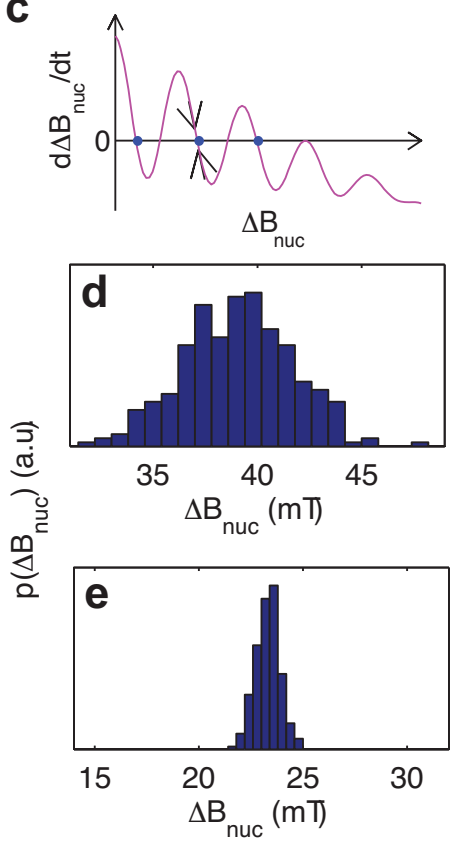

FIG. 7: Nuclear spin narrowing experiments in gate-defined quantum dots. (a) Schematic illustration of the pumping curve for ESR based feedback on a single electron $\operatorname{spin}^{45}$. Fluctuations away from the fixed points are compensated by a restoring pump effect (arrows). (b) Polarization dragging experiment for single electron spins using ESR. As the field increases from near zero, the singlet-triplet mixing is supressed while the CW-ESR signal is off resonant and has no effect. At $B_{e x t} \approx 50 \mathrm{mT}$, a resonance condition is met, thus breaking spin blockade. Subsequently, the resulting high current condition is maintained even while sweeping $B_{\text {ext }}$ up and down, thus demonstrating the locking of the total field $B_{e x t}+B_{n u c}$ to the resonance frequency: any change in $B_{e x t}$ is compensated by an opposite change in the nuclear polarisation. Figure is adapted from Ref. $^{45}$. (c) Pumping curve for voltage pulse based feedback on an $S-T_{0}$ qubit. The oscillatory structure arises from first letting the qubit precess in the hyperfine field and then executing a DNP step that is conditional on the state emerging from this evolution. (d) Distributions of the gradient nuclear field $\Delta B_{n u c}$ for fixed pumping, obtained by histogramming instantaneous values of $\Delta B_{n u c}$. The pumping produces a finite mean, but does not substantially change the width of the distribution. (e) Same measurement, but with feedback pumping applied between measurements, which narrows the distribution. Since the width includes a measurement uncertainty of the instantaneous value of $\Delta B_{z}$, the improvement of $T_{2}^{*}$ is larger than inferred from the distribution. Figures (c)-(e) are adapted from Ref. ${ }^{44}$. 
polarization rate as a function of the current value of the hyperfine field. Figs. 7(a),(c) show pumping curves for both feedback methods. A stable fixed point is obtained whenever the pumping curve crosses zero with a negative slope so that fluctuations away from the fixed point are corrected by an opposing pump effect.

The ESR pumping curve (Fig.7a) emerges from the resonance condition of the microwave excitation with the Zeeman field $B_{\text {ext }}+B_{n u c}$ seen by the electron, with the overall negative background slope arising from relaxation of the nuclear spin polarization ${ }^{45}$. The narrowing effect was inferred from dragging and locking of the ESR resonance frequency in response to changes of the externally applied field (Fig.7b), which were found to be compensated by nuclear polarization such that the total field remained constant ${ }^{45}$.

The oscillatory behavior of the corresponding pumping curve for the $S-T_{0}$ qubit (Fig.7c) was generated by initializing it in $S$ and then letting it precess between $S$ and $T_{0}$ for a time $\tau_{F B}$ under the influence of $\Delta B_{n u c}{ }^{44}$. Upon subsequently sweeping the gate voltages across the $S-T_{+}$transition, a nuclear spin can only be flipped by the $S$-component of the qubit state emerging from the evolution. Thus, the average pump rate is proportional to the $\Delta B_{n u c}$-dependent singlet probability. The stabilizing effect of this feedback scheme was demonstrated by measuring the $S-T_{0}$ qubit precession: narrowing of the $\Delta B_{n u c}$ distribution (Figs.7d,e) and corresponding enhancement of the qubit's $T_{2}^{*}$ from 16 ns to at least 150 ns were observed.

Note that it was reported earlier that the same type of DNP without feedback could extend $T_{2}^{*}$ to beyond $1 \mu \mathrm{s}^{90}$. Although theoretical scenarios ${ }^{91}$ have been proposed to explain such an effect ${ }^{90}$, it later turned out that another interpretation of the data is much more plausible ${ }^{92}$.

\section{Nuclear magnetic resonance in single quantum dots}

Direct manipulation of nuclear spins using nuclear magnetic resonance (NMR) is desirable for several reasons. As in the previous sections, this provides new insights in the spin properties of QD electrons and holes. NMR measurements provide information on the nuclear spin coherence, an important insight in the properties of the magnetic environment of the electron and hole spin-qubits. Pulsed NMR may also serve as a tool for fast redirection of the large Overhauser fields inside the dot, an additional tool for qubit control ${ }^{17}$. Finally, NMR can be used to reveal the structural properties of the dot to provide direct correlations with its electronic properties and feedback for QD fabrication.

The Hamiltonian for a nuclear spin $I$ having a gyromagnetic ratio $\gamma$ can be written as ${ }^{13,66}$ :

$$
H_{n u c}=-h \nu_{L} I_{z}+H_{Q}
$$

where $\nu_{L}=\gamma B_{z} /(2 \pi)$ is the nuclear Larmor frequency, $I_{z}$ the z-projection of the nuclear spin, and $H_{Q}$ describes the interaction of the nuclear quadrupole moment with the electric field gradient. $H_{Q}$ arises in quantum dots as a result of strain or alloy fluctuations, and is particularly pronounced in self-assembled QDs. In a magnetic resonance experiment transitions between spin states with $\Delta I_{z}= \pm 1$ are induced with a transverse magnetic field oscillating at a radio-frequency (rf) close to $\nu_{L}$. The corresponding changes in the nuclear spin state populations are detected using optical or electrical methods from changes in the electron Overhauser shift in QDs.

First NMR in QDs was carried out in optical measurements on single GaAs/AlGaAs interface dots ${ }^{12}$, where quadrupole effects were weak. The discrete exciton energy structure in QDs was successfully uti- 


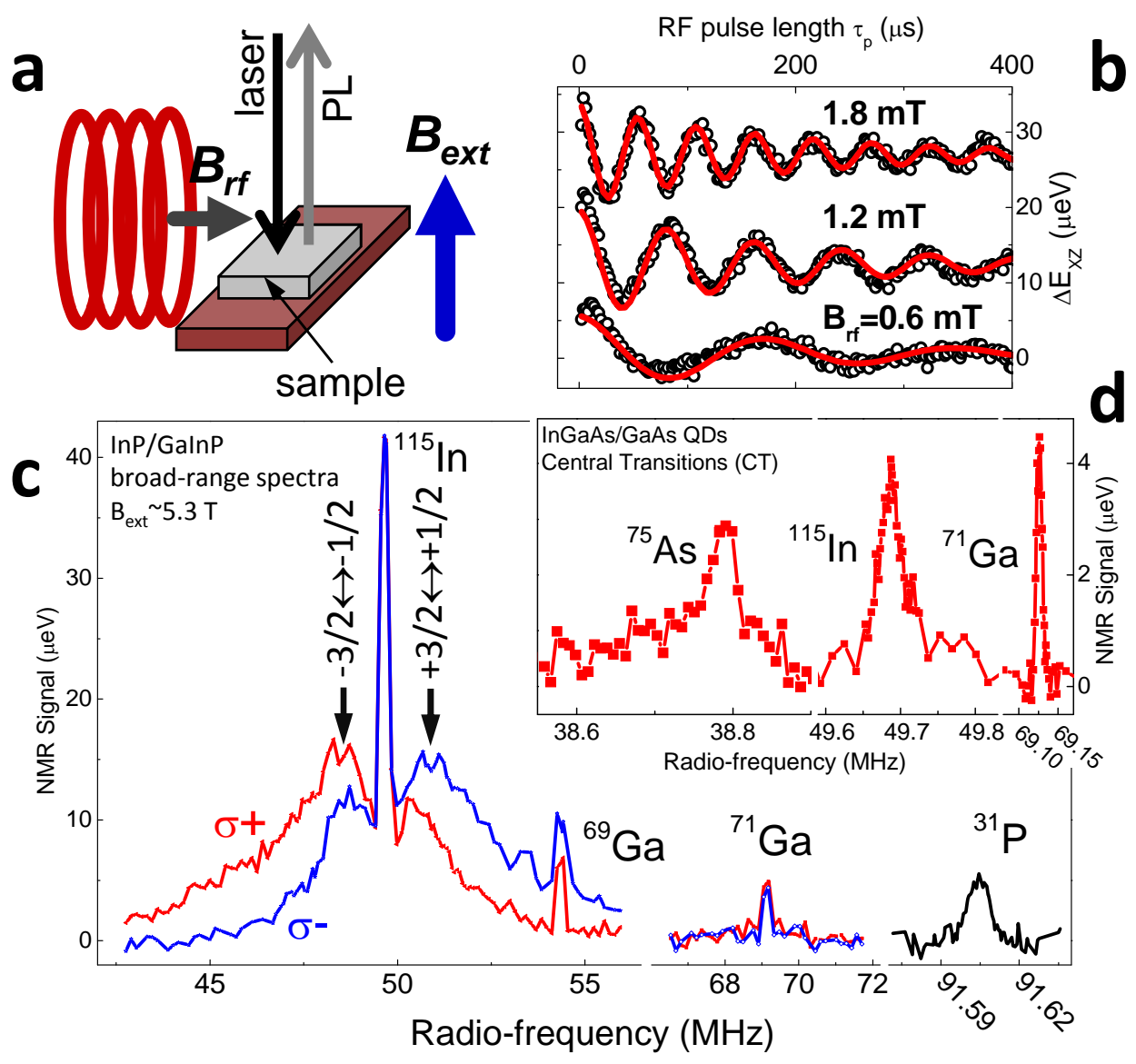

FIG. 8: Optically detected nuclear magnetic resonance (ODNMR) in single quantum dots. (a) Schematics of the ODNMR set-up, showing a mini-coil wound around a sample supplying an in-plane oscillating magnetic field $B_{r f}$. Optical excitation is along the growth axis of the dot and the external field, $B_{z}$. (b) Rabi oscillations of ${ }^{69} \mathrm{Ga}$ spins in a GaAs/AlGaAs QD measured for $B_{r f}=0.6,1.2$ and $1.8 \mathrm{mT}$ via the variation of the exciton Zeeman splitting as a function of the $\mathrm{rf}$ pulse duration $\left(B_{z}=3.55 \mathrm{~T}\right)$. Lines show fitting taking into account inhomogeneities of the nuclear Zeeman splitting still present in strain-free GaAs QDs. (c) Optically detected NMR spectra of strained self-assembled InP/GaInP quantum dots measured at $B_{z} \approx 5.3 \mathrm{~T}$ using the "inverse" method (see text) ${ }^{19}$ with $\sigma^{+}$(red curves) and $\sigma^{-}$(blue curves) optical pumping. For spin $1 / 2{ }^{31} \mathrm{P}$ unaffected by strain a saturation NMR spectrum (with single-frequency excitation) is shown. The sharp single peaks correspond to $-1 / 2 \leftrightarrow 1 / 2$ central transitions, weakly affected by strain with amplitudes proportional to isotope concentrations: significant substitution of indium by gallium in InP quantum dots is evidenced in (c) $\pm 3 / 2 \leftrightarrow \pm 1 / 2$ transitions least affected by strain (for $\left|I_{z}\right|>1 / 2$ ) give rise to secondary peaks (marked by vertical arrows). (d) High resolution "inverse" ODNMR spectra of central transitions measured in InGaAs QDs at $B_{z} \approx 5.3 \mathrm{~T} .{ }^{75} \mathrm{As}$ nuclei show greater sensitivity to strain compared with ${ }^{71} \mathrm{Ga}$ and ${ }^{115} \mathrm{In}$ resulting in a broader NMR line. Figures are adapted from Ref. ${ }^{17}$ in (b) and Ref. ${ }^{19}$ in (c) and (d). 
lized: changes in the electron Overhauser shift induced by rf excitation could be measured with accuracy of a few $\mu \mathrm{eV}$. NMR spectra were measured by simply stepping rf frequency through the resonance ${ }^{12,16}$. Further advancement of the nuclear spin control was made possible by employing pulsed NMR measured optically $^{17}$ in a single GaAs/AlGaAs QD and electrically ${ }^{18}$ in double GaAs/AlGaAs QD devices. Using such techniques the fast coherent rotations of nuclear spins under the influence of the rf and external field could be resolved, and the nuclear spin coherence time was measured ${ }^{17}$. Rabi oscillations, Ramsey fringes, spin-echo measurements and, finally, arbitrary rotations of large Overhauser fields about any axis on a time-scale of a few tens of micro-seconds were demonstrated ${ }^{17}$.

Techniques developed for strain-free GaAs dots have been found inapplicable in self-assembled QDs with strong quadrupole broadenings ${ }^{66}$ due to low NMR signal. Enhancement of NMR signal was achieved in large ensembles of QDs in transverse magnetic fields ${ }^{93,94}$. However, unambiguous interpretation of the experimental data was hampered by the complex spin dynamics ${ }^{95}$, further complicated by the presence of the strong quadrupole effects ${ }^{93,94}$.

Recently, high-resolution optically detected NMR has been carried out in single self-assembled InGaAs/GaAs and InP/GaInP QDs by employing novel spin population transfer techniques ${ }^{19}$. Instead of using a wide rf band in order to increase the number of affected nuclear spin transitions, an "inverted" rf spectrum was employed having two very broad bands ( $\approx 10 \mathrm{MHz}$ ) with a gap in between. This approach led to signal enhancement more than 100 for $9 / 2$ spins compared to the standard saturation techniques, and allowed measurements with resolution down to $\approx 10 \mathrm{kHz}^{19}$. These techniques reveal a wealth of structural information such as chemical composition and strain distribution in the volume of the wavefunction of the confined electron ${ }^{19}$, and present a powerful microscopy tool for non-invasive structural analysis of single QDs. In order to gain an additional enhancement in spatial resolution of NMR, the use of an effective magnetic (Knight) field of the photo-excited electron may be possible ${ }^{16}$. The spatial distribution of the Knight field follows that of the strongly localized electron wavefunction: Knight field gradients of the order of $10^{3} \mathrm{~T} / \mathrm{cm}$ can be achieved, potentially enabling determination of the nuclear spins position with resolution of $1 \mathrm{~nm}$ inside a single QD ${ }^{16}$.

\section{Future directions and other materials}

The above sections present the state-of-the-art in nuclear magnetism in semiconductor QDs. Below we comment on future developments in this field. We also briefly outline other classes of materials where electron-nuclear spin interactions have been investigated.

Control of nuclear spins for realization of coherent spin qubits. Efforts to achieve quiescent nuclear spins for improving coherence of the central spin (spin qubit) may continue in several directions. From analysis of QD composition using $\mathrm{NMR}^{19}$, it is now clear that polarization degrees of $90 \%$ or above may be accessible in optically pumped dots ${ }^{19,33}$. The effect of this on the coherence of electron or hole spin qubit needs to be verified. Alternatively, approaches achieving stabilized nuclear spin distributions are very attractive as they do not require very high polarization degrees.

Another way to achieve suppression of the nuclear spin fluctuations is to realize an ordered nuclear spin state $^{96}$. This in principle can be achieved by cooling nuclear spins to ultra-low sub- $\mu \mathrm{K}$ temperatures using adiabatic demagnetization (AD), although first attempts in self-assembled dots experienced difficulties 
owing to strong quadrupole effects ${ }^{65}$. In future, similar experiments could be attempted in unstrained GaAs dots.

Understanding of nuclear spin coherence in strained structures is another direction aimed at achieving quiescent magnetic environment. Recent initial studies showed more robust nuclear spin coherence in structures with strain ${ }^{97}$, also naturally present in self-assembled dots and some nanowires.

Holes remain rather attractive as a spin qubit due to the significantly weaker HI compared to that of electrons. Recent studies open the way for engineering of the hole-nuclear spin interaction by appropriate choice of QD composition ${ }^{87}$. In this way, improved hole spin qubit control may be obtained, a subject of further studies.

Nuclear spins: beyond the semi-classical approximation. In the coming years, we anticipate a new direction in research on quantum dots which takes nuclear spin control into the quantum regime. This is the regime where the nuclear spin state can no longer be captured in terms of a classical nuclear field or probability distribution of nuclear fields, which have been used to describe current experiments. Creating quantum states of the nuclear spin bath can be done using the coupling Hamiltonian between electron and nuclear spins, which implies that the electron will influence the dynamics of the nuclear bath via some quantum back action. It is thus very interesting to explore if there is an experimentally detectable deviation from classical models that can be unambiguously attributed to the back action effect.

As a first example that this may be possible, the creation of squeezed states of the nuclear spin bath in quantum dots was recently proposed, using microwave irradiation 98,99. In spin squeezing, the uncertainty of one component of the (total) spin is reduced below the uncertainty limit at the expense of increased uncertainty in an orthogonal component ${ }^{100}$. Interestingly, it was shown theoretically that sufficiently strong spin squeezing implies entanglement in the spin bath ${ }^{101}$. As another example, although harder to achieve with current techniques, proposals exist for coherent exchange of a qubit state between the quantum dot electron spin and a collective degree of freedom of the nuclear spin bath ${ }^{15}$. If realized, this would mean that the nuclear spin system can be used as a long-lived quantum memory, since even simple Hahn echo decay times of nuclear spins in quantum dots are about $1 \mathrm{~ms}^{17,18}$. Such a coherent information transfer would require special "dark" nuclear states with a reduced transverse hyperfine field, which in principle can be created via fast DNP. However, these states are highly sensitive to dephasing of the nuclear spins, and are subject to a fragile balance between hyperfine mediated spin transfer and dephasing due to the Knight shift ${ }^{102}$. A first step would thus be to establish whether such states, which would manifest themselves in a saturation of the nuclear polarization rate, can indeed be created. Another example is the creation of superradiance effects giving strongly enhanced electron-nuclear flip-flop rates, which could be observed in transport measurements ${ }^{103}$ as well as optical spectroscopy ${ }^{104}$. Common to all these examples is the collective effect of a large number of nuclear spins coherently interacting with a single (central) electron spin. Finally, it remains to be seen how much narrowing procedures can be improved, and whether they will eventually permit access to probe some form of intrinsic free induction decay that arises from the bath dynamics rather than ensemble averaging, as studied theoretically in Refs. ${ }^{74,79}$.

Other material systems. While this review focuses on III-V semiconducting quantum dots, there 
have been a handful of other material systems where the interplay between a central electron spin and the surrounding nuclear and even electron spin baths have been investigated. Prominent examples include carbon nanotubes ${ }^{105}$, both natural (with $1 \%$ of ${ }^{13} \mathrm{C}$ ) and $100 \%{ }^{13} \mathrm{C}$, phosphorus spins in $\mathrm{Si}^{106,107}$ and Si quantum dots ${ }^{108}$, and diamond NV centers ${ }^{109}$. While much of the physics discussed in this review is applicable to these systems, there are a few notable differences. For example the spin echo response of phosphorus spins in $\mathrm{Si}$ is due to the ${ }^{29} \mathrm{Si}$ host atoms and is theoretically and experimentally shown to have a time dependence given by $\exp \left[-\left(t / T_{2}\right)^{2.3}\right]$ (Refs. $\left.{ }^{78,106}\right)$. The resulting exponent of 2.3 as opposed to 4 in $\mathrm{GaAs}^{78,106}$ is a result of the detailed envelope wavefunctions associated with each system and is therefore not a universal exponent. A second example is recurrences in the electron spin echo signal as seen in Fig.5b. In GaAs such recurrences are a result of commensurate evolution of the nuclear spins of different species. A similar phenomenon is also seen in NV centers in diamond due to the ${ }^{13} \mathrm{C}$ nuclear spins. However, unlike the GaAs case where multiple species are required in order to see recurrences, in diamond, since the dominant interaction between the central spin and the nuclear spins is dipolar, a single nuclear spin species is sufficient.

\section{Acknowledgments}

E. A. C., M. N. M. and A. I. T. thank support by the EPSRC Programme grants (EP/G001642/1 and EP/J007544/1), ITN Spin-Optronics and the Royal Society. L. M. K. V. thanks support by a European Research Council starting grant, the Dutch Foundation for Fundamental Research on Matter (FOM), and the Office of the Director of National Intelligence, Intelligence Advanced Research Projects Activity (IARPA), through the Army Research Office grant W911NF-12-1-0354. H. B. thanks the Alfried Krupp von Bohlen und Halbach Foundation. K. C. N. acknowledges support from the Center for Probing the Nanoscale, an NSF NSEC, supported under grant no. PHY-0830228 and from NSF grant no. DMR-0803974.

* Now at: Department of Applied Physics, Stanford University, Stanford, CA 94305, USA

1 Petta, J. et al. Coherent manipulation of coupled electron spins in semiconductor quantum dots. Science 309, 2180-2184 (2005).

${ }^{2}$ Koppens, F. H. L. et al. Driven coherent oscillations of a single electron spin in a quantum dot. Nature 442, 766-771 (2006).

3 Brunner, D. et al. A Coherent Single-Hole Spin in a Semiconductor. Science 325, 70-72 (2009).

${ }^{4}$ Mikkelsen, M. H., Berezovsky, J., G., S. N., Coldren, L. A. \& Awschalom, D. D. Optically detected coherent spin dynamics of a single electron in a quantum dot. Nature Phys. 3, 770-773 (2007).

${ }^{5}$ Press, D. et al. Ultrafast optical spin echo in a single quantum dot. Nature Photon. 4, 367-370 (2010).

${ }^{6}$ De Greve, K. et al. Ultrafast coherent control and suppressed nuclear feedback of a single quantum dot hole qubit. Nature Phys. 7, 872-878 (2011).

7 Greilich, A., Carter, S. G., Kim, D., Bracker, A. S. \& Gammon, D. Optical control of one and two hole spins in interacting quantum dots. Nature Photon. 5, 703-709 (2011).

${ }^{8}$ Godden, T. M. et al. Coherent optical control of the spin of a single hole in an InAs/GaAs quantum dot. 
Phys. Rev. Lett. 108, 017402 (2012).

${ }^{9}$ Loss, D. \& DiVincenzo, D. P. Quantum computation with quantum dots. Phys. Rev. A 57, 120-126 (1998).

${ }^{10}$ Khaetskii, A. V., Loss, D. \& Glazman, L. Electron spin decoherence in quantum dots due to interaction with nuclei. Phys. Rev. Lett. 88, 186802 (2002).

11 Merkulov, I. A., Efros, A. L. \& Rosen, M. Electron spin relaxation by nuclei in semiconductor quantum dots. Phys. Rev. B 65, 205309 (2002).

12 Gammon, D. et al. Nuclear spectroscopy in single quantum dots: Nanoscopic Raman scattering and nuclear magnetic resonance. Science 277, 85-88 (1997).

13 Abragam, A. The principles of Nuclear Magnetism (Oxford University Press, London, 1961).

14 Meier, F. \& Zakharchenya, B. P. (eds.) Optical Orientation (Elsevier, New York, 1984).

15 Taylor, J. M., Marcus, C. M. \& Lukin, M. D. Long-lived memory for mesoscopic quantum bits. Phys. Rev. Lett. 90, 206803 (2003).

16 Makhonin, M. N. et al. Optically tunable nuclear magnetic resonance in a single quantum dot. Phys. Rev. B 82, 161309 (2010).

17 Makhonin, M. N. et al. Fast control of nuclear spin polarization in an optically pumped single quantum dot. Nature Mater. 10, 844-848 (2011).

18 Takahashi, R., Kono, K., Tarucha, S. \& Ono, K. Voltage-selective bidirectional polarization and coherent rotation of nuclear spins in quantum dots. Phys. Rev. Lett. 107, 026602 (2011).

19 Chekhovich, E. A. et al. Structural analysis of strained quantum dots using nuclear magnetic resonance. Nature Nanotech. 7, 646-650 (2012).

20 Tartakovskii, A. (ed.) Quantum Dots: Optics, Electron Transport and Future Applications (Cambridge University Press, Cambridge, 2012).

21 Lai, C. W., Maletinsky, P., Badolato, A. \& Imamoglu, A. Knight-field-enabled nuclear spin polarization in single quantum dots. Phys. Rev. Lett. 96, 167403 (2006).

22 Eble, B. et al. Dynamic nuclear polarization of a single charge-tunable InAs/GaAs quantum dot. Phys. Rev. B 74, 081306 (2006).

23 Tartakovskii, A. I. et al. Nuclear spin switch in semiconductor quantum dots. Phys. Rev. Lett. 98, 026806 (2007).

24 Braun, P.-F. et al. Bistability of the nuclear polarization created through optical pumping in InGaAs quantum dots. Phys. Rev. B 74, 245306 (2006).

25 Urbaszek, B. et al. Efficient dynamical nuclear polarization in quantum dots: Temperature dependence. Phys. Rev. B 76, 201301 (2007).

${ }^{26}$ Koppens, F. H. L., Nowack, K. C. \& Vandersypen, L. M. K. Spin echo of a single spin in a quantum dot. Phys. Rev. Lett. 100, 236802 (2008).

$27 \mathrm{Xu}$, X. et al. Optically controlled locking of the nuclear field via coherent dark-state spectroscopy. Nature 459, 1105-1109 (2009).

28 Bluhm, H. et al. Dephasing time of GaAs electron-spin qubits coupled to a nuclear bath exceeding $200 \mu \mathrm{s}$. Nature Phys. 7, 109-113 (2010).

29 de Sousa, R. \& Das Sarma, S. Theory of nuclear-induced spectral diffusion: Spin decoherence in phosphorus donors in Si and GaAs quantum dots. Phys. Rev. B. 68, 115322 (2003).

30 Yao, W., Lui, R.-B. \& Sham, L. J. Restoring coherence lost to a slow interacting mesoscopic spin bath. Phys. Rev. Lett. 98, 077602 (2007).

31 Shchukin, V. A. \& Bimberg, D. Spontaneous ordering of nanostructures on crystal surfaces. Rev. Mod. Phys. 71, 1125-1171 (1999).

32 Warburton, R. J. et al. Optical emission from a charge-tunable quantum ring. Nature 405, 926-929 (2000).

33 Chekhovich, E. A. et al. Pumping of nuclear spins by optical excitation of spin-forbidden transitions in a 
quantum dot. Phys. Rev. Lett. 104, 066804 (2010).

34 Bracker, A. S. et al. Optical pumping of the electronic and nuclear spin of single charge-tunable quantum dots. Phys. Rev. Lett. 94, 047402 (2005).

35 Maletinsky, P., Lai, C. W., Badolato, A. \& Imamoglu, A. Nonlinear dynamics of quantum dot nuclear spins. Phys. Rev. B 75, 035409 (2007).

36 Maletinsky, P., Badolato, A. \& Imamoglu, A. Dynamics of quantum dot nuclear spin polarization controlled by a single electron. Phys. Rev. Lett. 99, 056804 (2007).

37 Chekhovich, E. A., Krysa, A. B., Skolnick, M. S. \& Tartakovskii, A. I. Direct measurement of the hole-nuclear spin interaction in single InP/GaInP quantum dots using photoluminescence spectroscopy. Phys. Rev. Lett. 106, 027402 (2011).

38 Latta, C. et al. Confluence of resonant laser excitation and bidirectional quantum-dot nuclear-spin polarization. Nature Phys. 5, 758-763 (2009).

39 Fallahi, P., Yilmaz, S. T. \& Imamoğlu, A. Measurement of a heavy-hole hyperfine interaction in InGaAs quantum dots using resonance fluorescence. Phys. Rev. Lett. 105, 257402 (2010).

${ }^{40}$ Oulton, R. et al. Subsecond spin relaxation times in quantum dots at zero applied magnetic field due to a strong electron-nuclear interaction. Phys. Rev. Lett. 98, 107401 (2007).

41 Greilich, A. et al. Nuclei-induced frequency focusing of electron spin coherence. Science 317, 1896-1899 (2007).

${ }^{42}$ Hanson, R., Kouwenhoven, L. P., Petta, J. R., Tarucha, S. \& Vandersypen, L. M. K. Spins in few-electron quantum dots. Rev. Mod. Phys. 79, 1217-1265 (2007).

43 Hanson, R. \& Awschalom, D. D. Coherent manipulation of single spins in semiconductors. Nature 453, 1043-1049 (2008).

44 Bluhm, H., Foletti, S., Mahalu, D., Umansky, V. \& Yacoby, A. Enhancing the coherence of a spin qubit by operating it as a feedback loop that controls its nuclear spin bath. Phys. Rev. Lett. 105, 216803 (2010).

45 Vink, I. et al. Locking electron spins into magnetic resonance by electron-nuclear feedback. Nature Phys. 5, 764-768 (2009).

46 Nowack, K. C., Koppens, F. H. L., Nazarov, Y. V. \& Vandersypen, L. M. K. Coherent control of a single electron spin with electric fields. Science 318, 1430-1433 (2007).

47 Laird, E. A. et al. Hyperfine-mediated gate-driven electron spin resonance. Phys. Rev. Lett. 99, 246601 (2007).

48 Ono, K., Austing, D. G., Tokura, Y. \& Tarucha, S. Current rectification by pauli exclusion in a weakly coupled double quantum dot system. Science 297, 1313-1317 (2002).

49 Petta, J. R. et al. Dynamic nuclear polarization with single electron spins. Phys. Rev. Lett. 100, 067601 (2008).

50 Reilly, D., Marcus, C., Hanson, M. \& Gossard, A. Fast single-charge sensing with a rf quantum point contact. Appl. Phys. Lett. 91, 162101 (2007).

51 Barthel, C., Reilly, D., Marcus, C., Hanson, M. \& Gossard, A. Rapid single-shot measurement of a singlettriplet qubit. Phys. Rev. Lett. 103, 160503 (2009).

52 Baugh, J., Kitamura, Y., Ono, K. \& Tarucha, S. Large nuclear Overhauser fields detected in vertically coupled double quantum dots. Phys. Rev. Lett. 99, 096804 (2007).

53 Foletti, S., Bluhm, H., Mahalu, D., Umansky, V. \& Yacoby, A. Universal quantum control of two-electron spin quantum bits using dynamic nuclear polarization. Nature Phys. 5, 903-908 (2009).

54 Brown, S. W., Kennedy, T. A., Gammon, D. \& Snow, E. S. Spectrally resolved Overhauser shifts in single GaAs/AlGaAs quantum dots. Phys. Rev. B 54, R17339-R17342 (1996).

55 Kloeffel, C. et al. Controlling the interaction of electron and nuclear spins in a tunnel-coupled quantum dot. Phys. Rev. Lett. 106, 046802 (2011). 
56 Dyakonov, M. I. (ed.) Spin Physics in Semiconductors (Springer, 2008).

57 Makhonin, M. N. et al. Nuclear spin pumping under resonant optical excitation in a quantum dot. Appl. Phys. Lett. 93, 073113 (2008).

58 Klotz, F. et al. Asymmetric optical nuclear spin pumping in a single uncharged quantum dot. Phys. Rev. B 82, 121307 (2010).

59 Korenev, V. L. Nuclear spin nanomagnet in an optically excited quantum dot. Phys. Rev. Lett. 99, 256405 (2007).

${ }^{60}$ Latta, C., Srivastava, A. \& Imamoğlu, A. Hyperfine interaction-dominated dynamics of nuclear spins in self-assembled InGaAs quantum dots. Phys. Rev. Lett. 107, 167401 (2011).

61 Chekhovich, E. A. et al. Dynamics of optically induced nuclear spin polarization in individual inp/gainp quantum dots. Phys. Rev. B 81, 245308 (2010).

62 Makhonin, M. N. et al. Long nuclear spin polarization decay times controlled by optical pumping in individual quantum dots. Phys. Rev. B 77, 125307 (2008).

63 Nikolaenko, A. E. et al. Suppression of nuclear spin diffusion at a GaAs/AlGaAs interface measured with a single quantum-dot nanoprobe. Phys. Rev. B 79, 081303 (2009).

64 Dzhioev, R. I. \& Korenev, V. L. Stabilization of the electron-nuclear spin orientation in quantum dots by the nuclear quadrupole interaction. Phys. Rev. Lett. 99, 037401 (2007).

65 Maletinsky, P., Kroner, M. \& Imamoglu, A. Breakdown of the nuclear-spin-temperature approach in quantumdot demagnetization experiments. Nature Phys. 5, 407-411 (2009).

66 Bulutay, C. Quadrupolar spectra of nuclear spins in strained $\operatorname{In}_{x} \mathrm{Ga}_{1-x}$ As quantum dots. Phys. Rev. B 85, 115313 (2012).

67 Belhadj, T. et al. Controlling the polarization eigenstate of a quantum dot exciton with light. Phys. Rev. Lett. 103, 086601 (2009).

68 Ono, K. \& Tarucha, S. Nuclear-spin-induced oscillatory current in spin-blockaded quantum dots. Phys. Rev. Lett. 92, 256803 (2004).

69 Koppens, F. H. L. et al. Control and detection of singlet-triplet mixing in a random nuclear field. Science 309, 1346-1350 (2005).

70 Rudner, M. S. \& Levitov, L. S. Electrically driven reverse Overhauser pumping of nuclear spins in quantum dots. Phys. Rev. Lett. 99, 246602 (2007).

71 Rudner, M. S., Koppens, F. H. L., Folk, J. A., Vandersypen, L. M. K. \& Levitov, L. S. Nuclear spin dynamics in double quantum dots: Fixed points, transients, and intermittency. Phys. Rev. B 84, 075339 (2011).

72 Danon, J. et al. Multiple nuclear polarization states in a double quantum dot. Phys. Rev. Lett. 103, 046601 (2009).

73 Cywiński, L., Witzel, W. M. \& Das Sarma, S. Electron spin dephasing due to hyperfine interactions with a nuclear spin bath. Phys. Rev. Lett. 102, 057601 (2009).

74 Coish, W. A., Fischer, J. \& Loss, D. Exponential decay in a spin bath. Phys. Rev. B 77, 125329 (2008).

75 Reilly, D. J. et al. Measurement of temporal correlations of the Overhauser field in a double quantum dot. Phys. Rev. Lett. 101, 236803 (2008).

76 Reilly, D. et al. Exchange control of nuclear spin diffusion in a double quantum dot. Phys. Rev. Lett. 104, 236802 (2010).

77 Hahn, E. L. Spin echoes. Phys. Rev. 80, 580 (1950).

78 Witzel, W. M. \& Das Sarma, S. Quantum theory for electron spin decoherence induced by nuclear spin dynamics in semiconductor quantum computer architectures: Spectral diffusion of localized electron spins in the nuclear solid-state environment. Phys. Rev. B 74, 035322 (2006).

79 Yao, W., Lui, R.-B. \& Sham, L. J. Theory of electron spin decoherence by interacting nuclear spins in a quantum dot. Phys. Rev. B 74, 195301 (2006). 
${ }^{80} \mathrm{Li}$, Y. et al. Intrinsic spin fluctuations reveal the dynamical response function of holes coupled to nuclear spin baths in (In,Ga)As quantum dots. Phys. Rev. Lett. 108, 186603 (2012).

81 Heiss, D. et al. Observation of extremely slow hole spin relaxation in self-assembled quantum dots. Phys. Rev. B 76, 241306 (2007).

82 Gerardot, B. D. et al. Optical pumping of a single hole spin in a quantum dot. Nature 451, 441-444 (2008).

${ }^{83}$ Gryncharova, E. I. \& Perel, V. I. Relaxation of nuclear spins interacting with holes in semiconductors. Sov. Phys. Semicond. 11, 997 (1977).

84 Testelin, C., Bernardot, F., Eble, B. \& Chamarro, M. Hole-spin dephasing time associated with hyperfine interaction in quantum dots. Phys. Rev. B 79, 195440 (2009).

85 Fischer, J., Coish, W. A., Bulaev, D. V. \& Loss, D. Spin decoherence of a heavy hole coupled to nuclear spins in a quantum dot. Phys. Rev. B 78, 155329 (2008).

86 Eble, B. et al. Hole-nuclear spin interaction in quantum dots. Phys. Rev. Lett. 102, 146601 (2009).

87 Chekhovich, E. A. et al. Element-sensitive measurement of the hole-nuclear spin interaction in quantum dots. Nature Phys. 9, 74-78 (2013).

88 Desfonds, P. et al. Electron and hole spin cooling efficiency in InAs quantum dots: The role of nuclear field. Appl. Phys. Lett. 96, 172108 (2010).

89 Glazov, M. M., Yugova, I. A. \& Efros, A. L. Electron spin synchronization induced by optical nuclear magnetic resonance feedback. Phys. Rev. B 85, 041303 (2012).

90 Reilly, D. J. et al. Suppressing spin qubit dephasing by nuclear state preparation. Science 321, 817-821 (2008).

91 Ribeiro, H. \& Burkard, G. Nuclear state preparation via Landau-Zener-Stückelberg transitions in double quantum dots. Phys. Rev. Lett. 102, 216802 (2009).

92 Barthel, C. et al. Relaxation and readout visibility of a singlet-triplet qubit in an Overhauser field gradient. Phys. Rev. B 85, 035306 (2012).

93 Flisinski, K. et al. Optically detected magnetic resonance at the quadrupole-split nuclear states in InGaAs/GaAs quantum dots. Phys. Rev. B 82, 081308 (2010).

94 Cherbunin, R. V. et al. Resonant nuclear spin pumping in InGaAs quantum dots. Phys. Rev. B 84, 041304 (2011).

95 Krebs, O. et al. Anomalous hanle effect due to optically created transverse Overhauser field in single InAs/GaAs quantum dots. Phys. Rev. Lett. 104, 056603 (2010).

96 Simon, P., Braunecker, B. \& Loss, D. Magnetic ordering of nuclear spins in an interacting two-dimensional electron gas. Phys. Rev. B 77, 045108 (2008).

97 Kondo, Y. et al. Multipulse operation and optical detection of nuclear spin coherence in a GaAs/AlGaAs quantum well. Phys. Rev. Lett. 101, 207601 (2008).

98 Rudner, M. S., Vandersypen, L. M. K., Vuletić, V. \& Levitov, L. S. Generating entanglement and squeezed states of nuclear spins in quantum dots. Phys. Rev. Lett. 107, 206806 (2011).

99 Kessler, E. M. et al. Dissipative phase transition in a central spin system. Phys. Rev. A 86, 012116 (2012).

100 Kitagawa, M. \& Ueda, M. Squeezed spin states. Phys. Rev. A 47, 5138-5143 (1993).

101 Sørensen, A. S. \& Mølmer, K. Entanglement and extreme spin squeezing. Phys. Rev. Lett. 86, 4431-4434 (2001).

102 Gullans, M. et al. Dynamic nuclear polarization in double quantum dots. Phys. Rev. Lett. 104, 226807 (2010).

103 Schuetz, M. J. A., Kessler, E. M., Cirac, J. I. \& Giedke, G. Superradiance-like electron transport through a quantum dot. Phys. Rev. B 86, 085322 (2012).

104 Kessler, E. M., Yelin, S., Lukin, M. D., Cirac, J. I. \& Giedke, G. Optical superradiance from nuclear spin environment of single-photon emitters. Phys. Rev. Lett. 104, 143601 (2010). 
105 Kuemmeth, F., Churchill, H. O. H., Herring, P. K. \& Marcus, C. M. Carbon nanotubes for coherent spintronics. Materials Today 13, 18-26 (2010).

106 Abe, E. et al. Electron spin coherence of phosphorus donors in silicon: Effect of environmental nuclei. Phys. Rev. B 82, 121201 (2010).

107 Morton, J. J. L. et al. Solid-state quantum memory using the ${ }^{31} \mathrm{P}$ nuclear spin. Nature 455, 1085-1088 (2008).

108 Shaji, N. et al. Spin blockade and lifetime-enhanced transport in a few-electron Si/SiGe double quantum dot. Nature Phys. 4, 540-544 (2008).

109 Dutt, M. V. G. et al. Quantum register based on individual electronic and nuclear spin qubits in diamond. Science 316, 1312-1316 (2007). 